\title{
Cataract surgery programmes in Africa
}

\section{T Y Wong}

\section{Towards evidence based public health}

$\mathrm{R}$ eaders of the $B J O$ will be aware that cataract is the leading cause of blindness in Africa, affecting an estimated half of the seven million blind people in that continent. ${ }^{1}$ This number is likely to increase substantially, as approximately 600000 Africans become blind from cataract each year. Despite the enormity of the problem, few cataract operations are performed in Africa. The cataract surgery rate (CSR), a measure of the volume of cataract surgery performed in a population, is about 500 per million per year in Africa. To tackle cataract blindness, the Vision 2020 initiative, a programme involving the World Health Organization, the International Agency for Prevention of Blindness, and various governmental and non-governmental organisations, aims to increase the CSR in Africa to about 2000 per million per year. ${ }^{2}$

Substantial progress has been made on several fronts over the past few years. There has been a steady stream of information on the epidemiology, distribution, and impact of cataract in different parts of Africa. ${ }^{34}$ There is also increasing evidence that well designed and well executed cataract surgery programmes can provide effective high volume and high quality cataract surgical services in selected communities in Africa. ${ }^{5}$

Despite encouraging signs, there remain significant challenges and barriers. Thus, there may be important lessons in the few "successful" cataract surgery programmes that emerge from Africa. In this month's issue of the BJO (p 1237), Lewallen and colleagues share their experience of two such programmes, both of which appeared to have contributed towards a significant increase in the number of cataract surgeries performed in their communities. Since the introduction of these programmes, the authors estimate that CSR have risen to 1583 per million in the Kwale District in Kenya, and 1165 per million in the Kilimanjaro Region in Tanzania in 2004. These are remarkable figures, all the more so given that previous estimates of the CSR were approximately 644 per million in
Kenya and 313 per million in Tanzania in 2002.

What were the key lessons learnt from these "successful" cataract surgery programmes? The authors describe several. These include a close link between the community screening programmes and the hospital services that provide the cataract surgery services, a seamless "patient friendly" referral chain, the utilisation of a community screening examination team that has appropriate ophthalmic expertise to identify people most likely to benefit from cataract surgery, and selecting populations that both need the services and have the ability to sustain such programmes. None of these concepts is very new, of course. Indeed, many of the features described in this article have been proposed in other African communities, and apply elsewhere in other developing countries (for example, India). ${ }^{6}$

\section{Like changes in clinical practice resulting from a heightened aware- ness of evidence based medicine, evidence based public health is necessary in ensuring the major paradigm changes needed in public health practice}

A unique aspect of this study was using a quantitative outcome (in this case, the CSR) to qualify the success for the programme, information that many previous descriptive studies have lacked. ${ }^{7}$ None the less, there are several limitations in this study that may serve as areas for future research. For example, the authors argue in their article that one of the key lessons of the cataract surgery programme was to "make specific changes at the hospital essential to providing more service." A list of these changes is described in the article. However, the study lacked more quantitative analyses on the specific changes described, which may make it difficult to translate these features to other programmes. Future studies should more precisely quantify the specific features or processes of a programme, and whether one feature or process is independent of and, more important, relative to others. Secondly, although the article attributes the high CSR in the two communities to the introduction of the cataract surgery programmes, it would have been vastly more instructive to have data on the actual change in CSR over time (that is, before and after the introduction of the programme).

Some of this information will not be readily available, given the resources and situation. Indeed, we recognise that evaluating the effectiveness of cataract surgery programmes is challenging enough, even in the most ideal circumstances in developed Western countries. However, it is crucial for public health ophthalmology to move away from purely descriptive, experience based case studies towards evidence based public health.

What is evidence based public health? It is a variation of evidence based medicine, focusing instead on a public health intervention. ${ }^{8}$ Evidence based public health seeks to answer several questions regarding the usefulness of an intervention. ${ }^{9}$ Firstly, has the research performed been sufficiently valid to support a decision on whether or not to implement an intervention (in this case, a particular cataract surgery programme)? Secondly, have all the important research outcomes been evaluated (CSR is one example, post-cataract surgery visual outcome would be another)? Thirdly, is the research applicable to the potential recipients of the intervention (would such cataract surgery programme be transferable to other African communities and settings)? Although these questions are similar in many respects to the evaluation of evidence in medicine, there are some differences. Compared to a treatment in clinical medicine, public health interventions tend to be multifaceted, more complex, and more dependent on specific situations. ${ }^{10}$ Thus, it is more critical to distinguish between the efficacy of an intervention, and the effectiveness of an intervention in terms of delivery and execution.

Evidence based public health is but one factor in the process of translating a research observation into broad policy decisions regarding the implementation of a particular intervention. Like changes in clinical practice resulting from a heightened awareness of evidence based medicine, evidence based public health is necessary in ensuring the major paradigm changes needed in public health practice. For public health ophthalmology, it will be the first step towards the elimination of avoidable blindness from cataract in Africa and other developing countries. 
Br J Ophthalmol 2005;89:1231-1232.

doi: $10.1136 /$ bjo. 2005.072645

Correspondence to: Tien Yin Wong, MD, PhD Centre for Eye Research Australia, University of Melbourne, 32 Gisborne Street, Victoria 3002, Australia; twong@unimelb.edu.au

Competing interests: none declared

\section{REFERENCES}

1 Lewallen S, Courtright P. Blindness in Africa: present situation and future needs. Br J Ophthalmol 2001;85:897-903.
2 Foster A. Cataract and "Vision 2020-the right to sight" initiative. Br J Ophthalmol 2001;85:635-7.

3 Congdon N, West SK, Buhrmann RR, et al. Prevalence of the different types of age-related cataract in an African population. Invest cataract in an African population. Invest
Ophthalmol Vis Sci 2001;42:2478-82.

4 Rabiu MM. Cataract blindness and barriers to uptake of cataract surgery in a rural community of northern Nigeria. $\mathrm{Br} J$ Ophthalmol northern Nigeria.

5 Limburg H, Foster A, Gilbert C, et al. Routine monitoring of visual outcome of cataract surgery. Part 2: Results from eight study centres. Br J Ophthalmol 2005;89:50-2.

6 Natchiar G, Robin AL, Thulasiraj RD, et al. Attacking the backlog of India's curable blind. The
Aravind Eye Hospital model. Arch Ophthalmol 1994; 112:987-93

7 Rotchford AP, Rotchford KM, Mthethwa LP, et al. Reasons for poor cataract surgery uptake-a qualitative study in rural South Africa. Trop Med Int Health 2002;7:288-92.

8 Heller RF, Page J. A population perspective to evidence based medicine: "evidence for population health". J Epidemiol Community Health 2002;56:45-7.

9 Kohatsu ND, Robinson JG, Torner JC. Evidencebased public health: an evolving concept. J Prev Med 2004;27:417-21.

10 Rychetnik L, Frommer M, Hawe P, et al. Criteria for evaluating evidence on public health interventions. J Epidemiol Community Health 2002;56:119-27.

TRACHOMA

\section{Trachoma}

\section{J D Chidambaram, T M Lietman}

\section{A tale of two diseases}

$\mathrm{T}$ rachoma is in a sense two diseases: the infection most apparent in children and the blinding sequelae found in adults. Ocular strains of Chlamydia trachomatis cause repeated episodes of conjunctivitis, with the peak prevalence of infection usually occurring in 3-5 year olds. ${ }^{1}$ Progressive scarring, entropion, and trichiasis lead to blinding corneal ulceration, typically some 40 years or more later. ${ }^{2}$ This long lag time between infection and blindness has always been somewhat of a puzzle. Several explanations have been offered. The normal, age related decrease in tears, goblet cells, and lid elasticity may make the elderly more prone to the effects of scarring. ${ }^{3}$ Existing scars may contract slowly over time. Further episodes of chlamydial infection may cause scarring to progress. ${ }^{45}$ If this last hypothesis is the case, then the frequent recurrence of trichiasis after surgical repair could in part be because of recurrent infection. This is a testable hypothesis. A single dose of oral azithromycin is effective in eliminating chlamydial infection in an individual, ${ }^{6}$ and treatment given to a community can offer a sustained reduction of infection even a year later. ${ }^{7}$ With some effort, infection can be prevented, at least for the year or so after surgery.

In this issue of the BJO (p 1282), Burton et al report a trial in which they randomised patients receiving trichiasis surgery into two groups. One group received topical tetracycline postsurgery as standard of care. The other received topical tetracycline and, in addition, they and their household members received a dose of oral azithromycin at the time of surgery and 6 months later. Recurrence of trichiasis 12 months after surgery proved to be a fairly common event, providing the study with some power to detect a difference. However, recurrence was equally frequent in the two arms. Also, chlamydial infection was not a risk factor for recurrence.

This trial makes us think about trachoma differently, even though azithromycia could not be shown to prevent recurrence of trichiasis after surgery

Although the authors could not implicate chlamydia in the failure of trichiasis surgery, secondary analyses suggest that other bacteria may have a role. Identification of a pathogenic species of bacteria colonising the conjunctiva was a twofold risk factor for recurrence of severe trichiasis. Bacterial colonisation may just be a surrogate marker for more severe scarring, which itself is a risk factor for recurrence. However, it is also possible that bacterial infections themselves cause progression of disease. This would not be the first time that nonchlamydial bacteria have been implicated in trachoma, ${ }^{8}$ but it might be the most convincing evidence so far. Colonisation of the conjunctiva has also been found in other cicatricial conjunctivitides, such as ocular cicatricial pemphigoid (OCP). In fact, corneal ulceration in OCP is often caused by the same species of bacteria that had colonised the conjunctiva on previous visits. ${ }^{9}$ In Western countries, prophylactic antibiotic drops are sometimes provided to patients at such a high risk for corneal ulcers. Burton et al's results suggest that maybe we should consider such prophylaxis in trachoma patients after trichiasis surgery.

This was a well designed and well organised trial. Strengths include a large number of patients, a large number of surgeons, good follow up, and excellent microbiology. However, it may not be the last word on the importance of chlamydia in the progression of cicatricial trachoma. Azithromycin is already being distributed to everyone in endemic areas; some may argue that extra doses in older, postsurgical patients are hardly necessary since infection in adults is less common, ${ }^{1}$ of a shorter duration, ${ }^{10}$ and a lower load. ${ }^{11}$ On the other hand, estimates of this progression are higher in areas with more infection (Tanzania) than they are in areas with less infection (Gambia). ${ }^{12-14}$ Two other groups are examining this question with somewhat similar designs (Emily West, Sheila West, Wondu Alemayehu, and Deborah Dean, personal communications). One trial is being performed in an area of Ethiopia where trachoma is hyper-endemic and the opportunity for re-infection far greater. These studies may or may not produce similar results.

This trial makes us think about trachoma differently, even though azithromycin could not be shown to prevent recurrence of trichiasis after surgery. Currently, programs treat trachoma as two different diseases, reducing infection with antibiotics and eliminating trichiasis with surgery. Burton et al tested whether the link between the two was stronger than appreciated. Their bacteriological results may even suggest an entirely new front in trachoma control. The question of how cicatricial trachoma will progress in the absence of recurrent chlamydial infections is an important one. Even if mass antibiotic distributions and other measures are successful in dramatically 
reducing infection in entire populations, there will still remain a generation of individuals with scarred conjunctivae. In some areas, we may no longer have to contend with two trachomas, only the cicatricial form. It would have been comforting had this trial found that trichiasis would not recur in the absence of chlamydia. Hopefully, further research will optimise the management of cicatricial trachoma.

Br J Ophthalmol 2005;89:1232-1233. doi: 10.1136/bjo.2005.067322

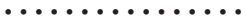

Authors' affiliations

J D Chidambaram, T M Lietman, FI Proctor Foundation, University of California, San Francisco, CA, USA

T M Lietman, Institute for Global Health, and Department of Ophthalmology, University of California, San Francisco, CA, USA
Correspondence to: Dr Thomas M Lietman, WHO Collaborating Center, FI Proctor Foundation, Room 307, 95 Kirkham Street, University of California San Francisco, San Francisco, CA 94143-0944, USA tml@itsa.ucsf.edu

\section{REFERENCES}

1 Bird M, Dawson CR, Schachter JS, et al. Does the diagnosis of trachoma adequately identify ocular chlamydial infection in trachoma-endemic areas? $J$ Infect Dis 2003;187:1669-73.

2 Mabey DC, Solomon AW, Foster A. Trachoma. Lancet 2003;362:223-9.

3 Bowman RJ, Jatta B, Cham B, et al. Natural history of trachomatous scarring in the Gambia: results of a 12-year longitudinal follow-up. Ophthalmology 2001;108:2219-24.

4 Zhang H, Kandel RP, Sharma B, et al. Risk factors for recurrence of postoperative trichiasis: implications for trachoma blindness prevention. Arch Ophthalmol 2004;122:51 1-16.

5 West ES, Mkocha H, Munoz B, et al. Risk factors for postsurgical trichiasis recurrence in a trachoma-endemic area. Invest Ophthalmol Vis Sci 2005;46:447-53.
6 Bailey RL, Arullendran P, Whittle HC, et al Randomised controlled trial of single-dose azithromycin in treatment of trachoma. Lance 1993:342:453-6.

7 Schachter J, West SK, Mabey D, et al. Azithromycin in control of trachoma. Lancet 1999;354:630-5.

8 Jones BR. The prevention of blindness from trachoma. Trans Ophthalmol Soc UK 1975:95:16-33.

9 Holsclaw DS. Ocular cicatricial pemphigoid. Int Ophthalmol Clin 1998;38:89-106.

10 Bailey R, Duong T, Carpenter R, et al. The duration of human ocular Chlamydia trachomatis infection is age dependent. Epidemiol Infect 1999; 123:479-86

11 Solomon AW, Holland MJ, Burton MJ, et al. Strategies for control of trachoma: observational study with quantitative PCR. Lancet 2003;362:198-204.

12 Muñoz B, Bobo L, Mkocha H, et al. Incidence of trichiasis in a cohort of women with and without scarring. Int J Epidemiol 1999:28:1167-71.

13 Bowman RJ, Faal H, Myatt M, et al. Longitudinal study of trachomatous trichiasis in the Gambia. Br J Ophthalmol 2002;86:339-43.

14 Lietman T. Trachoma control: the beginning of the end? Ophthalmology 2001;108:2163-4.

\section{Utility analysis tells all}

\section{Brown}

\section{We must embrace it and foster its use}

$\mathrm{t}$ is most gratifying to see the excellent article by Gupta et al in this issue of

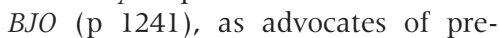
ference based quality of life instruments such as utility analysis (those that ask patients to make a choice about the desirability of their quality of life) versus function based instruments (those that measure primarily function: physical, psychological, vocational, avocational, cognitive, social, and so forth). ${ }^{1}$

Why are we such fans of utility analysis? The answer lies in the definition of what is the most desirable quality of life instrument. ${ }^{1}$ A good instrument should be:

(1) all encompassing with regard to the variables that comprise quality of life

(2) sensitive to small changes in health

(3) reliable (reproducible)

(4) applicable across all medical specialties

(5) able to be completed within a reasonable time period

(6) able to be understood by patients, and

(7) able to demonstrate construct validity (the ability to measure what it is intended to measure
(8) applicable for us in performing healthcare economic analyses.

Utility analysis meets all of these parameters and is a major pillar of value based medicine, the practice of medicine that incorporates the best evidence based data with patient preferences to assess the value conferred by our interventions, whether medical, surgical, and/or pharmaceutical. Of special note as well is the fact that these patient derived utility values that measure the quality of life associated with health states appear to be innate to human nature. How do we know? Because data $^{23}$ suggest that, for the most part, utility values transcend sex, ethnicity, age, level of education, income, and even nationality, There are, of course, exceptions, such as this analysis found with education, but overall human attitudes towards illness are remarkably similar.

Utility analysis incorporates improvements in quality of life conferred by interventions that can be integrated with improvement in length of life to quantify the total value gained from the interventions. This value (measured using the QALY, or quality adjusted life year) is comparable across every intervention in health care. The most frequently utilised quality of life instruments in ophthalmology, the VF14 and the NEI-VFQ-25, are not especially applicable across all specialties and have not been used in healthcare economic analyses.

As policy makers and other stakeholders in health care utilise quality of life data to make allocation decisions, it seems natural that they would favour an instrument than encompasses all interventions in health care, rather than just ophthalmology, which accounts for a small fraction of the healthcare dollar. ${ }^{1}$

How should we, as ophthalmologists, approach the introduction of value based medicine? With open arms. The great majority of ophthalmic interventions confer extraordinary value, and it is value that patients want. An added benefit is that the conferred value is great compared to the costs expended. Cataract surgery in the United States costs $\$ 600-\$ 700 /$ QALY. It is a bargain by any measure.

Br J Ophthalmol 2005;89:1233.

doi: 10.1136/bjo.2005.074294

Correspondence to: Melissa Brown, Center for Value-Based Medicine, PO Box 335 Flourtown PA 19031-1404, USA; mbrown@ valuebasedmedicine.com

\section{REFERENCES}

1 Brown MM, Brown GC, Sharma S. Evidencebased to value-based medicine. Chicago: AMA Press, 1995: 1-324.

2 Brown MM, Brown GC, Sharma S, et al. Health care economic analyses and value-based medicine. Surv Ophthalmol 2003;48:204-23.

3 Brown MM, Brown GC, Sharma S, et al. Utility values associated with blindness in an adult population. Br J Ophthalmol 2001;85:327-31. 